(C) Astles, Cariad, Fisher, Emma, Purcell-Gates, Laura, Sextou, Persephone, 2020. The definitive, peer reviewed and edited version of this article is published in Journal of Applied Arts and Health,11, 1,200-207, 2020, https://doi.org/10.1386/jaah_00037_5

\title{
Broken Puppet Symposia
}

\section{Cariad Astles, Emma Fisher, Laura Purcell-Gates, Persephone Sextou}

The Broken Puppet symposia took place over three years: 2017, 2018 and 2019. Our reflections on and review of these symposia follow below.

\section{Broken Puppet 1}

The first Broken Puppet symposium took place on $1^{\text {st }}$ and $2^{\text {nd }}$ August 2017 at University College, Cork. It was a collaboration between the UNIMA Research Commission, Mary Immaculate College Limerick, University College Cork and the Cork puppetry festival and was led by Emma Fisher and Cariad Astles. We have written about the choice of title for this symposium, and subsequent symposia, in the editorial to this journal volume. Broken Puppet 1 was the first international meeting of academics and practitioners working specifically on the relationships between puppetry, healthcare, wellbeing and disability, and has led to further collaborations, research enquiries and, ultimately, to this publication itself. Being the first of its nature, the symposium attracted considerable international attention, hosting talks, performances and presentations from people from the following countries: Ireland, the UK, Spain, Finland, Chile, Germany, Costa Rica, Japan and Brazil.

The symposium was intended to examine theory and practice within the fields of puppetry, disability and healthcare, and papers were invited which considered embodied puppetry practices; breaking the body's borders; a consideration of what puppetry does to concepts of normativism in body-based terms; methods of inclusivity: participatory puppetry in healthcare contexts; puppetry, illness, disability and identity; making the invisible visible; and healthcare interventions using puppetry.

There were two keynote speakers, both contributors to this volume: Dr Melissa Trimingham from the University of Kent, UK, and Dr Persephone Sextou from Newman University, 
Birmingham, UK. The two days were divided into four panels, each dealing with a specific area of research: these panels were: wellbeing; disability; hospitals and care settings, and mental health.

On $1^{\text {st }}$ August the symposium was introduced by Cariad Astles, president of the UNIMA Research Commission. Melissa Trimingham then gave the first keynote on the AHRC-funded project 'Imagining Autism', a research investigation into ways in which puppetry can benefit young people on the autistic spectrum. The wellbeing panel which followed included presentations by Moira Jenkins from Ireland, with a position paper discussing puppetry as a human right; Andrea Markovits, a teacher, puppeteer and therapist, on puppetry therapy and traumatic memory in Chile post-dictatorship; Yasuko Senda, a puppetry scholar, director and promoter, on the use of puppets to promote social wellbeing in Japan; Oscar Goldszmidt on social inclusion through puppetry in Brazil, through a case study of someone with cerebral palsy; Caroline Astell-Burt, from the UK, on closeness, touching and kinaesthesia and Antje Wegener, a puppet therapist from Germany. The first day closed with a discussion panel which explored the wide and considerable ways in which puppetry can be used as a means to activate communitas, social bonding, positive relational encounters and indeed, promote and advocate for human rights.

Day 2, the $2^{\text {nd }}$ August, began with the second keynote speech from Persephone Sextou from Newman University, on her extensive work using puppetry in hospitals, clinics and healthcare settings, through the CADLab (Community and Applied Drama Laboratory) project based at Newman University. The second panel of the symposium explored puppetry in relation to disability. The following speakers presented, the first two from Ireland and the third from Brazil: Emma Fisher, on her PhD project exploring her own identity through the relationship between puppetry and disability; Corina Duyn on her own practice and journey with puppetry and Roberto Ferreira Da Silva on his educational work using puppetry with disabled participants.

Figure 1: Research practice based on Pupa by Emma Fisher. Ceramic puppet made in collaboration with Sheila Stone, 2017, Photo Emma Mac.

The second panel of the day focused on puppetry in, and for use within healthcare centres. The speakers were Riku Laakkonen from Finland, who discussed the use of puppets as 'performing objects' in palliative care; their links to memory, purpose and identity and Matt Jennings from Northern Ireland, who leads a project in training medics and emergency care 
workers in practicing care and handling of 'other' bodies, through the use of life-sized mannequins. The project includes training in puppetry by Karen Torley of Banyan Puppet Theatre and encourages the students to consider the precious and fragile nature of the body that they are handling, as a meaningful life, and not just as a physical body to be moved from place to place. The symposium was then joined by Gibdel Wilson, an emergency doctor from Costa Rica, who uses puppets to communicate with children in emergency situations and in end-of-life care. All the speakers commented on puppetry's ability to communicate with the vulnerable, and to engender care, through the imaginative process undergone when the puppet or object takes on personality.

The final panel of the symposium comprised a combination of performances and presentations on the theme of puppetry and mental health. Here, similarly to some of the talks presented within the disability panel, scholars and practitioners of puppetry testified to puppetry as a powerful means to express what cannot be expressed in other contexts; to reconfigure and understand identity, and to build tools towards acceptance of self. One of the speakers stated that at an earlier date, confined to a mental health unit, puppetry literally saved their life, through providing a means to visualise and understand their own mental health condition and to trace a pathway through the understanding of self in relation to the world. The speakers in the final panel included three from the UK and one from Spain. Marisa Latimer spoke about her work using shadow puppetry as dramatherapy; using the symbolic and imagery-laden framework of shadows for her participants to disentangle difficult or traumatic events; Kate James-Moore, who spoke about puppetry as a creative tool within mental health contexts; Joni-Rae Carrack, who explored the relationship between objectivity and subjectivity in puppetry; and Aaron Jean Crombé, from Spain, who used puppetry as a metaphor for self-acceptance in wider contexts of mental health.

The final reflections were given by puppeteer Lesley Burton who summed up the exciting work presented and noted the combination of practice-based research and academic content; the unique nature of the symposium itself and the great enthusiasm for its continuation; the importance of the so-far-under-researched and theorised work, and the variety and richness of the papers presented.

Broken Puppet 1 demonstrated that there was great interest in and thirst for a space to discuss puppetry within the contexts noted above. The event was energetic, positive and drew 
attention, firstly, to the previous lack of any suitable fora to explore ideas, theories and approaches, and secondly, to the great potential for this work to develop.

\section{Broken Puppet 2}

The second Broken Puppet symposium took place at Bath Spa University on $14^{\text {th }}$ and 15 th April 2018 and was led by Laura Purcell-Gates. Having been inspired by the idea of Broken Puppet 1, Purcell-Gates, in conversation with Emma Fisher, proposed to bring a closer focus within Broken Puppet 2 on the ways in which puppetry and disability intersect to produce innovative art forms. The symposium also wished to include workshops which would enable the participants to extend some of the previous thinking into practical sharings of work. The event was co-sponsored by the Arts and Social Change Research Group at Bath Spa University, the UNIMA Research Commission, and Puppet Place in Bristol.

Broken Puppet 2 was framed as a creative combination of panels, roundtables, workshops, presentations of practice, and practice-based 'laboratories' in which intersections of disability performance and puppetry would be intensively explored. The intention behind this structuring was to tap into the creative potential of having disability performance artists, puppeteers and artists who traversed these fields together in the same room, exploring the issues together. Foregrounding the voices of disabled artists and scholars was a particular priority of the symposium.

The first day of the symposium began with a welcome from organisers Laura Purcell-Gates and Emma Fisher, followed by the first keynote by Corina Duyn. Duyn, who first presented her work at Broken Puppet 1, is a puppetry artist and writes for Disability Arts Online. Duyn took the audience through her experiences of 'life interrupted' by an M.E. diagnosis, followed by a creative reinvention of her life and work as an artist, reflecting on the ways working with puppets has enabled her to artistically explore the embodied experience of living with illness. 
This was followed by presentations of practice from Pablo Ariel who spoke about the work of Galilee Deaf Theatre; Nikki Charlesworth who presented her own puppets constructed and puppeteered to reflect her experiences of disability; and Emma Fisher who spoke about her doctoral thesis that culminated in her show Pupa, a process that was about claiming her disabled identity and exploring the constructs of the disabled body.

Puppeteer Chris Pirie from Puppet Place began the afternoon sessions with an overview of a series of events on puppetry and disability that had been hosted at the 2017 Bristol Festival of Puppetry. During this 10-day festival, Diverse City, an organisation working for diversity in the arts, and Extraordinary Bodies, an integrated circus company, had facilitated workshops and labs to explore intersections of disability and puppetry; Guerilla Dance Project and OpenUp Music had conducted a lab to develop eye gaze assisted puppetry; and Hijinx Theatre had worked with puppetry companies Blind Summit and Green Ginger to explore tabletop puppetry with learning disabled adults. These diverse performance-based projects engaged with and provoked areas of inquiry around the ways in which bodies and social spaces can be understood and performed differently, and inspired the symposium's laboratory sessions.

The first afternoon session consisted of three panels: one on 'Disability and puppetry in performance' featuring David Grant, Matt Hargrave and Paul McNamara; one on 'Applied puppetry and health' featuring Marina Tsaplina, Karl Tizzard-Kleister and Riku Laakkonen; and one on 'Puppetry and other(ed) identities' featuring Melissa Trimingham, Matt Smith and Kate James-Moore. The themed panels allowed a focus for presenters to share work within sub-themes of puppetry and disability performance, provoked discussion and raised questions to be taken into the labs.

Two labs followed the panels: 'Disabled puppet building' facilitated by Nikki Charlesworth, Chris Pirie and Emma Fisher; and 'Objects and bodies: exploring puppetry and disability performance through material-centred techniques' facilitated by Matt Smith and Laura Purcell-Gates. The lab format was open and exploratory, allowing questions and ideas generated in the first part of the day to be put into practice, and producing additional topics for discussion and exploration to be taken into day two of the symposium.

The evening session of the first day featured a presentation by disability artist and culture activist and scholar Petra Kuppers reading 'Disability culture fantasies', her recently- 
published short story collection Ice Bar. Kuppers described the event as 'an evening of lush embodiment and queer/crip storytelling.'

The second day of the symposium began with an introduction to UNIMA by Cariad Astles, followed by Petra Kuppers' keynote speech, titled: 'There are disabled people in the future: non-realist embodiment and puppetry'. Kuppers took her audience through an extended meditation on moments in which puppets have enacted non-realist embodiment in disability settings, including a deep consideration of the puppetry in Patrick Elkin's Cthulhu Stories at Dreamland Theatre in Michigan.

This was followed by a lab titled 'Breakdancing puppets: knowledge co-creation with puppetry and special schools' facilitated by Amelia De-Felice and Karian Schuitema, and a workshop titled 'For my eyes contain ten dances': using puppetry as a dramatherapy medium with trauma victims, facilitated by Daniel Stolfi of The Awesome Puppet Company.

The afternoon included a roundtable titled 'Lab results' in which symposium participants reflected on the knowledge, ideas, questions and provocations arising from the labs, and a final wrap-up discussion that included generating ideas for moving forward with this work.

\section{Broken Puppet 3}

Astles, Fisher and Purcell-Gates were joined for Broken Puppet 3 by Persephone Sextou, who proposed to host the third symposium at Newman University in Birmingham. The event was jointly organised by the Community and Applied Drama Laboratory (CADLab) and Newman University with the support of the UNIMA Research Commission. Following Broken Puppet 1 and Broken Puppet 2, the organisers decided that there was a clear need to discuss training in this area. Broken Puppet 3 was therefore titled and themed: 'Puppetry: Community, Health, Wellbeing and Disability: professional training opportunities'. Broken Puppet 3 took place at Newman University on $17^{\text {th }}$ and $18^{\text {th }}$ April 2019.

CADlab, in conjunction with UNIMA, recognised the need to introduce into curricula a stronger applied puppetry training dimension and to encourage universities, acting schools and puppetry schools to play their part in raising awareness of puppetry in relation to applied theatre. Broken Puppet 3 provided the opportunity for academics, artists and health 
professionals from 11 different countries (Finland, France, Italy, Ireland, Germany, Greece, Lebanon, Switzerland, Netherlands, the UK and the USA) to come together and share their perspectives on training. Contributors explored theoretical approaches and practical ways of using puppetry, object theatre, shadow theatre and toys in applied theatre, to address social issues and problems of individuals of all age groups and communities in health and wellbeing contexts and environments. There were two keynote presentations from Professor Ross Prior from the University of Wolverhampton, and Raven Kaliana, Director of (R)Evolution Puppet theatre company. Prior addressed the question of training and which elements of crossdisciplinary training should, or could, be included for practitioners and students of puppetry within these social contexts. Kaliana spoke from her own traumatic childhood experiences, and of her journey into making puppetry work, and the experience of making this work widely accessible, as part of wider social policy training initiatives. On the first day, contributors Melissa Trimingham from the UK, Katriina Andrianov and Riku Laakkonen from Finland, Marina Tsaplina from the USA, Matt Smith from the UK, Karim Dakroub from Lebanon and Becky Davies from the UK presented perspectives from their own work linking training opportunities with specified groups within the community and raising key questions about training itself: who for? Where can it take place? How can it retain accessibility? During the afternoon sessions, workshops were presented by Laura PurcellGates from the UK/USA, Karl Tizzard-Kleister and Karen Torley, from Ireland/Germany; by Annemarie Hänni-Reber, Esther Koller-Duse and Corinne Michel-Kund from Switzerland, and by Nenagh Watson from the UK. The workshops introduced participants to aspects of puppetry work with questions of disability, therapy, fragility, and in nursing education. The day ended with a performance, 'Love versus Trauma', by keynote speaker Raven Kaliana. On day two, after the keynote speech by Ross Prior, papers were shared by Cariad Astles from the UK, Emma Fisher from Ireland, Antje Wegener from Germany and Persephone Sextou, on puppetry as love, care and caress; on disability perspectives; on the therapeutic process and on models of intervention in hospitals.

The afternoon of $18^{\text {th }}$ April continued with workshops led by Daniel Stolfi (UK), Meg Amsden (UK); Amy Franczak (UK); Frans Hakkemars (Netherlands); Gilbert Meyer (France) and Mayra Stergiou (UK/Greece) on geographies of health and wellbeing, working with the elderly, on dramatherapy; on shadow theatre with those with dementia, on intergenerational work with puppetry and on working with the wellbeing of migrants. 
The afternoon continued with a sharing of artistic practice by Theresa McNally, Tzella Karali, Wesley Rolston and Kim Roberts, all from the UK, discussing work in dementia care and pupil referral units and of the role of story, narrative, ownership and empowerment through the object.

Broken Puppet 3, as was its remit, ended with the drafting a Manifesto on professional training of applied puppeteers in contexts of healthcare, wellbeing and disability. The top five areas noted were:

1. Puppetry is inherently interdisciplinary and so can 'meet' any other discipline. Its power lies at the interdisciplinary intersection of psychology, sociology, pedagogy and the arts.

2. Applied puppetry has the potential to use emergent technologies and robotics and work with the intersection between technology and health.

3. A puppet is not a puppet without its puppeteer; without a human being holding, caring, and intending it; training in emotional skills is therefore important.

4. Applied puppetry training has to include open access workshops, but fundamental theoretical input is needed for rigour and understanding.

5. Several training options could be possible, such as short courses; more academic courses; training aimed at practising professionals; diploma, seminar and placement courses; postgraduate training.

The organisers collected feedback on Broken Puppet 3 by questionnaire. Participants were impressed by the progression from theory to practice and found the content 'insightful', 'motivational' and 'inspiring', particularly enjoying the mixture of theory and practice. For the majority of participants, the focus of Broken Puppet 3 on training, health care and social improvement was a much-needed topic, but others were keen to maintain the focus on disability.

Reflecting on all three Broken Puppet Symposia retrospectively reassures us in thinking that the joined powers of performing arts, puppetry, theatre, and creative therapies can foster creativity, imagination, collaboration, and can teach us how to be more caring, kind and compassionate to ourselves and others in times of personal, social and global crisis. 
